\title{
The Principle of Certainty
}

\section{Muhammad Yasin}

Savar, Dhaka, Bangladesh

E-mail:md.yasin@ymcontents.com

1 Abstract: It is possible to prove the principle of certainty in a different way from the theory of relativity, de Broglie particle wave duality, the principle of uncertainty. The principle of certainty is the formula for measuring the position and momentum of a particle at the same time. If the theory of relativity, the principle of uncertainty is correct, then the principle of certainty must be considered correct. Because it is possible to prove the principle of certainty from the principle of uncertainty, it is also possible to prove the principle of certainty from the theory of special relativity. Again the idea of the principle of certainty comes from the de Broglie particle wave duality principle. Max Planck's radiation formula can be proved from the principle of certainty.

2 Keywords : uncertainty principle, quantum certainty mechanics, quantum measurement, simultaneous measurement, certain measurement

3 Introduction: Accepting the concept of uncertainty reveals the clarity of the principle of certainty. From the Uncertainty principle, if we consider the position - momentum $(\Delta x \Delta p)$ as a packet of certain value, the certainty principle can be proved by multiplying an additional uncertain value $(\Delta \mathrm{k})$ to respect the uncertainty principle. If a particle is expressed through a special theory of relativity, then the principle of certainty can be proved according to the theory of relativity. According to the theory of relativity, the momentum and position of a particle can be accurately measured by Lorentz conversion when the particle travels a certain distance (x), with momentum (p).Again the principle of certainty can also be proved from the de Broglie particle wave duality principle. When a particle moves with $\mathrm{p}$ momentum, the involved wave $(\lambda)$ is revealed. If the position of a particle is at a distance $(x)$ after time $(t)$, then the principle of certainty can be proved by applying the particle wave dual formula of de Broglie between distance and time. The principle of certainty applies to large particles ranging from atomic particles. Certainty principles can accurately predict the past and future of particles.

4 Keywords: quantum mechanics; uncertainty principle; certainty mechanics ; quantum measurement

\section{Method:}

5.1 Method (1)-The Principle of certainty from de Broglie's wave particle duality : 
Heisenberg's uncertainty principle shows that the position and momentum of a particle cannot be measured accurately at the same time. The more accurately we measure one, the more uncertain the other will become. Einstein thought that uncertainty was not entirely right. There is a mistake that has led to uncertainty. The particles follow some secret rule. I will now extract that secret rule from this principle of certainty. One thing we have noticed in Heisenberg's formula is that light has to be emitted on electrons to determine their position. In order to determine the position accurately, it is necessary to emit high frequency light that means more light. But we are not bringing this matter in calculation, we are saying that light of $\lambda$ wavelength has to be shed. Light is a 'photon' particle. If the photon falls on the electron, it will push the electron with " $F$ " force. That is, light of $f$ frequency will push the electron. But we are not doing that calculation, we can accurately determine the momentum and position by calculating the external impact in the laboratory. If we bring $\mathrm{f}$ frequency light in the calculation then the "certainty principle" will come.

The momentum of the particle and the wavelength of the involved wave from The Broglie's equation;

$\lambda=\frac{\mathrm{h}}{\mathrm{mv}}$

therefore,

$\mathrm{p}=\frac{\mathrm{h}}{\mathrm{c}} \mathrm{f}$

From equation 1 it is clear that when an object moves at a p momentum, waves are involved due to its momentum. Now imagine, an electron is moving at "p" momentum. The momentum of electrons will create a wave that will have a frequency $\mathrm{f}$.

Suppose, if the electron moves with velocity $\mathrm{v}$, crosses $\mathrm{x}$ distance at $\mathrm{t}$ time then the equation will be

$\mathrm{x}=\mathrm{vt}$

Now when the electron moves at a p momentum, the frequency of the wave (involved wave) will be created due to the momentum of the electron according to Equation 1 . From the duality of The Broglie we know that waves are intertwined with electrons. Electrons have a velocity. The wave involved with the electron also has a velocity. The velocity of an electron is called the particle velocity and the wave velocity of the wave associated with the electron is called the phase velocity. But the problem is that the velocity of the wave attached to the electron is not equal to the velocity of the electron. The phase velocity is $\mathrm{Va}=\mathbf{f} \boldsymbol{\lambda}$. And the velocity of the particle is $\mathbf{V}$

$\mathrm{V}=\frac{\mathrm{h}}{\mathrm{m} \lambda}$

The velocity of a particle and the velocity of a wave associated with a particle are not equal, but the velocity of a particle is equal to the velocity of a bunch of waves associated with a particle.

Cluster velocity is - many waves travel in clusters when they are transmitted in the same direction. The velocity of this cluster wave is called cluster velocity. suppose cluster velocity is $\mathrm{Vg}$.

$V_{g}=\frac{d \omega}{d k}$ 
The relationship between cluster velocity and phase velocity

$\mathrm{V}_{\mathrm{g}}=\mathrm{V}_{\mathrm{a}}-\lambda \frac{d v(a)}{d \lambda}$

Now the velocity of the particle and the velocity of the cluster are equal

So, If $\mathbf{V g}=\mathbf{V}$

$\mathrm{V}=\mathrm{V}_{\mathrm{a}}-\lambda \frac{\mathrm{dv}(\mathrm{a})}{\mathrm{d} \lambda}$

Cluster velocity and particle velocity are equal but particle velocity and wave velocity are not equal. So Va will not be equal to $\mathrm{V}$. But in this case a work can be done. Let's imagine the currencies of America and Bangladesh. According to the current market, 1 US dollar is equal to 85 Bangladeshi take. The value of 1 dollar is not 1 taka but one can represent another currency. What can be done with 10 dollars can be done with 850 taka.

How far you can go with 1 dollar by car you can go the same distance in the same country with 85 taka.

$\mathrm{t}=$ taka

$\mathrm{T}=$ dollar

$\mathrm{V}=$ car's velocity

Now if I go with taka by car then I can write

$\mathrm{S}=\mathrm{Vt}$

Or if I go with dollar by car then I can write

$\mathrm{S}=\mathrm{VT}[\mathrm{T} \neq \mathrm{t}$ but we can represent $\mathrm{t}$ by $\mathrm{T}]$

(Compared here with two currencies of the same country)

It turns out that money or dollars can go the same distance. So we can represent $t$ instead of T. In exactly the same way we can represent the velocity of a particle with the velocity of a wave.

So here we put the value of $\mathrm{Va}$ instead of velocity $\mathrm{v}$ at $\mathrm{S}=\mathrm{vt}$.

$\mathrm{V}_{\mathrm{a}}=\mathrm{f} \lambda$

From 2 no. and 7 no. equation-

$\mathrm{x}=\mathrm{f} \lambda \mathrm{t}$

$$
\begin{aligned}
& \Rightarrow \mathrm{x}=\frac{\mathrm{fh}}{\mathrm{p}} \quad\left[\lambda=\frac{\mathrm{h}}{\mathrm{p}}\right] \\
& \Rightarrow \mathrm{xp}=\mathrm{hft} \\
& \therefore \mathrm{xp}=\mathrm{hft}
\end{aligned}
$$

Equation 8 is the fourth law of quantum certainty mechanics, the "principle of certainty". By this formula it is possible to accurately determine the momentum and position of an object.

\subsection{Method (2) - principle of certainty according to the theory of relativity:}

$\mathrm{xp}=\mathrm{hft}$

The above certainty principle formula is a combination of Newton's formula. Now let us see the proof of the principle of certainty according to the special relativity theory.

Suppose your size is equal to an electron. You will go to Dhaka bus stop in a car at fixed velocity. The car you are sitting in is shaped like a hydrogen atom. Your friend is also shaped like an electron and he is standing $\mathrm{x}$ meters away from the bus stop. You are going to the bus stop at v velocity. After a while your distance from the bus stop is $\mathrm{x}$ meters. That means at that moment you and your friend are at the same distance from the bus stop. The distance and time of the two are being calculated from this very moment. Now the bus stop is $\mathrm{x}$ meters away from 
you and the bus stop is x meters away from your friend. Since then the time for the two of you has started from 00. Your friend is fixed so his position will not change over time. The distance between your friend and the bus stop is $\mathrm{x}$ meters .The distance between you and the bus stop will decrease $x^{\prime}$ over time as you are going at fixed velocity. The distance between you and the bus stop,

$\mathrm{x}^{\prime}=\mathrm{x}-\mathrm{vt}$

Now the speed of your car may be closer to the speed of light. So according to Lorentz's conversion,

$$
\begin{aligned}
X^{o_{1}} & =\frac{x_{1}-v t}{\sqrt{1-\frac{v^{2}}{c^{2}}}} \\
& \Rightarrow X^{o_{1}} m_{0}=\frac{m_{o}}{\sqrt{1-\frac{v^{2}}{c^{2}}}}\left(x_{1}-v t\right) \\
& \Rightarrow X^{o_{1}} m_{0}=m\left(x_{1}-v t\right) \quad\left(m=\frac{m_{o}}{\sqrt{1-\frac{v^{2}}{c^{2}}}}\right) \\
& \Rightarrow X^{o_{1}} m_{0}=m x_{1}-m v t
\end{aligned}
$$

$\mathrm{X}^{\mathrm{o}}{ }_{1} \mathrm{~m}_{\mathrm{o}}+\mathrm{mvt}=\mathrm{mx}_{1}$

Multiply by $\mathrm{C}$ on both sides of Equation (9).

$$
\begin{aligned}
& \mathrm{X}^{\mathrm{o}_{1}} \mathrm{~m}_{\mathrm{o}} \mathrm{c}+\mathrm{mvct}=\mathrm{x}_{1} \mathrm{mc} \\
& \Rightarrow \mathrm{X}^{\mathrm{o}}{ }_{1} \mathrm{~m}_{\mathrm{o}} \mathrm{c}+\mathrm{px}=\mathrm{x}_{1} \mathrm{mc} \quad[\mathrm{mv}=\mathrm{p}, \mathrm{ct}=\mathrm{x}]
\end{aligned}
$$

Now the bus is going at fixed velocity so,

$\mathrm{t}=\frac{\mathrm{x}}{\mathrm{v}}$

so,

$$
\begin{array}{ll}
\mathrm{X}^{\mathrm{o}_{1}} \mathrm{~m}_{\mathrm{o}} \mathrm{c}+\mathrm{px}=\mathrm{mc} \frac{\mathrm{x}}{\mathrm{v}} \mathrm{v} & \\
\Rightarrow \mathrm{X}^{\mathrm{o}}{ }_{1} \mathrm{~m}_{\mathrm{o}} \mathrm{c}+\mathrm{px}=\mathrm{pct} & {\left[\mathrm{t}=\frac{\mathrm{x}}{\mathrm{v}}, \mathrm{p}=\mathrm{mv}\right]} \\
\Rightarrow \mathrm{X}^{\mathrm{o}}{ }_{1} \mathrm{~m}_{\mathrm{o}} \mathrm{c}+\mathrm{px}=\mathrm{Et} & {[\mathrm{E}=\mathrm{pc}]}
\end{array}
$$

Therefore,

$\mathrm{X}^{\mathrm{o}_{1}} \mathrm{~m}_{\mathrm{o}} \mathrm{c}+\mathrm{px}=\mathrm{hft} \quad[\mathrm{E}=\mathrm{hf}]$

Now the bus was at fixed velocity so,

$\mathrm{X}_{1}=\mathrm{vt}$

$X_{1}-v t=0$

From equations (9) and (11) we get,

$\mathrm{X}^{\mathrm{o}_{1}}=\frac{\mathrm{x}_{1}-\mathrm{vt}}{\sqrt{1-\frac{\mathrm{v}^{2}}{\mathrm{c}^{2}}}}$ 
$\mathrm{X}^{\mathrm{o}_{1}}=0 \quad\left[\mathrm{x}_{1}-\mathrm{vt}=0\right]$

We get from Equation (10), "

$\mathrm{X}^{\mathrm{o}_{1}} \mathrm{~m}_{\mathrm{o}} \mathrm{c}+\mathrm{px}=\mathrm{hft}$

$\Rightarrow \mathrm{px}=\mathrm{hft} \quad\left[\mathrm{Xo}_{1}=0\right]$

Therefore,

$\mathrm{px}=\mathrm{hft}$

Equation 12 is the "principle of certainty" which is the fourth law of quantum certainty mechanics.

5.3 Method (3) Certainty principle From the principle of uncertainty:

Suppose we have two numbers $\mathrm{p}$ and $\mathrm{q}$. We do not know the exact value of $\mathrm{p}$ and $\mathrm{q}$ but we assume that the value of $p$ and $q$ is 10 or greater than 10.The higher the value of $p$ and $q$, the more accurate $p$ and $q$ will be but there is no set value. To say more value can be infinite value But then there will be problems. So we assume for the convenience of calculation that if the value of $\mathrm{p}$ and $\mathrm{q}$ is close to 10 then it will be close to the correct value. Now we are given an equation. That is the product of $\mathrm{p}$ and $\mathrm{q}$ is 10 i.e. $\mathrm{pq}=10$.From this equation, if we get closer to the correct value of $\mathrm{p}$, we will move away from the correct value of $\mathrm{q}$. Again, if we get close to the correct value of $\mathrm{q}$, we will move away from the correct value of $\mathrm{p}$. That is, if you know one value, the value of the other will be wrong. The value of the product of $p$ and $q$ can be 10 in many ways,

$\mathrm{pq}=0$

$10 \times 1=10$

or , $5 \times 2=10$

or, $1 \times 10=10$

or, $2 \times 5=10$

Our equation is,

$\mathrm{pq}=10$

From this equation we will never get the value of $p=10$ and the value of $q=10$ at the same time. But if we multiply an additional constant by pq then the values of $p$ and $q$ will be 10 at the same time. Suppose the correct value of $p$ is 10 and the correct value of $q$ is 10 .Then $p q k=10$ according to the above equation. That is, if $p=10, q=10$ then $k=1 / 10$. Now if the exact value of $p$ and $q$ is close to 10 then the value of $\mathrm{k}$ will go towards uncertainty. If we consider $\mathrm{p}$ and $\mathrm{q}$ as a fixed value then the value of $\mathrm{k}$ will be uncertain. According to the above equation, the product of two packets should be 10 , where pq is a packet of fixed value and the remaining indefinite value $\mathrm{k}$ is another packet.

(pq)k 10

In this way we will express Heisenberg's principle of uncertainty. According to uncertainty the values of momentum $\mathrm{p}$ and position $\mathrm{x}$ can never be measured with certainty at the same time. If the momentum is confirmed the position will become uncertain. The indefinite product of the two states will be equal to or greater than the Planck constant that is $\Delta \mathrm{p} \Delta \mathrm{x}=\hbar$. Now, according to the uncertainty principle, even if one has a value, one has to be uncertain. Now suppose by any means we know $\Delta \mathrm{p}$ and $\Delta \mathrm{x}$ for sure. Whether by observing it or in some other way. Suppose we were able to invent an instrument that could accurately measure the position $(\Delta x)$ and 
momentum $(\Delta \mathrm{p})$ of a particle. Then an additional constant $\mathrm{k}$ has to be multiplied to protect the uncertainty principle. $\mathrm{k}$ is an ongoing uncertainty value that has been used to protect the uncertainty principle. If $\Delta \mathrm{x} \Delta \mathrm{p}$ is confirmed then the constant $\mathrm{k}$ will be an uncertain value. Then we can write the equation,

$\Delta \mathrm{p} \Delta \mathrm{xk}=\hbar$

$\mathrm{k}$ is an unknown constant. If $\mathrm{k}=1$ then the uncertainty formula will be $\Delta \mathrm{x} \Delta \mathrm{p}=\hbar$. The definite value of momentum and position has been taken as a packet.

Then the equation will be,

$(\Delta \mathrm{x} \Delta \mathrm{p}) \times \mathrm{k}=\hbar$.

One packet is of momentum-position and the other packet is of constant $\mathrm{k}$.

Now if $\mathrm{k}$ is confirmed $(\Delta \mathrm{x} \Delta \mathrm{p})$ will be uncertain. That is, if $\mathrm{k}=1$ is confirmed $(\Delta \Delta \mathrm{p})$ will be uncertain. Again, if $(\Delta x \Delta p)$ is confirmed, $\mathrm{k}$ will be uncertain. Now suppose, we are sure $(\Delta x \Delta p)$ then the value of $\mathrm{k}$ is uncertain according to uncertainty.

From $(\Delta \mathrm{x} \Delta \mathrm{p}) \times \mathrm{k}=\hbar$ the uncertainty of energy and time will be:-

We know kinetic energy $\mathrm{E}=\frac{1}{2} \mathrm{~m} \mathrm{v}^{2}$.

Now We get by Taking the first derivative of the total E (i. e. kinetic energy) with respect $\mathrm{p}$, $\Delta \mathrm{E}=\frac{p}{m} \Delta \mathrm{p}$

$\Delta \mathrm{E}=\mathrm{v} \Delta \mathrm{p}$

We get from Equation 14 and Equation 13,

$\Delta \mathrm{E} \Delta \mathrm{xk}=\mathrm{v} \hbar$

$=>(\Delta \mathrm{E} \Delta \mathrm{t}) \mathrm{k}=\hbar$

From the above equation 15 we can see that imagining energy-time $(\mathrm{E} \Delta \mathrm{t})$ as a fixed packet will make the $\mathrm{k}$ constant uncertain.

Equation 13 is divided by Equation 14.

$\frac{(\Delta \mathrm{x} \Delta \mathrm{p}) \times \mathrm{k}=\hbar}{(\Delta \mathrm{E} \Delta \mathrm{t}) \mathrm{k}=\hbar}$

In equation $16(\Delta \mathrm{x} \Delta \mathrm{p})$ and $(\Delta \mathrm{E} \Delta \mathrm{t})$ are definite values . so we can write,

$\frac{(\mathrm{xp}) \times \mathrm{k}=\hbar}{(\mathrm{E} \Delta \mathrm{t}) \mathrm{k}=\hbar}$

Since Equation 17 comes from Equation 13, the value of $\Delta \mathrm{k}$ is the same in both cases.

So we can write,

$\mathrm{xp}=\mathrm{Et}$

Therefore,

$\mathrm{xp}=\mathrm{hft}$

Equation 18 is the principle of certainty which comes from the principle of uncertainty. 
From equation 18 position $(\mathrm{x})$, momentum $(\mathrm{p})$, time $(\mathrm{t})$, frequency $(\mathrm{f})$ are the definite values. Thus, knowing the momentum and frequency, the position can be determined after $(\mathrm{t})$ time. The more accurately the momentum can be measured, the more certain the position will be.

$\mathrm{xp}=\mathrm{hft}$ is the principle of certainty by which after knowing the frequency of the waves involved in the electron if we know the momentum we will be able to know the position, and if we know the position we will be able to know the momentum. When we go to see the particle in the laboratory and hit it with a photon, the particle receives hf amount of energy. If we ignore that, we will not be able to know the position and momentum of the particle at the same time. Electrons are particles like a marble(toy) and photons are also particles like a marble(toy).If you hit the marble with the marble, the speed-position is known by calculating the strength of the hit. Similarly, when looking at an electron in a laboratory, it is important to bring in the hf amount of energy in the calculation due to the $\mathrm{F}$ force of the photon. In the above equation hf amount of energy is brought And the momentum-position of the particle can be known simultaneously after the $\mathrm{t}$ time from the equation. $\mathrm{xp}=\mathrm{hft}$; this equation is established by maintaining the condition of uncertainty from the principle of uncertainty.

6 Decision: The principle of certainty is one of the most important formulas of quantum mechanics that can explain the cause of uncertainty in the principle of uncertainty. The past and future of a particle can be determined using the certainty principle $\mathrm{xp}=\mathrm{hft}$. When the particle moves with the p momentum, the wavelength $(\lambda)$ is revealed. Knowing the frequency $f$ of the wave, it is possible to accurately measure the momentum-position (xp) of the particle over time. The more accurately we can determine the position of a particle, the more accurately we can determine the momentum of a particle at the same time, if we can measure the frequency (f) and time $(\mathrm{t})$ of the waves involved with the particle.

7 Conclusion: The principle of certainty is a formula by which Max Planck's quantum theory can be explained, Bohr's angular momentum formula with uncertainty principle can be proved. Recently I have published two research papers named " Quantum Certainty Mechanics" and " Why Does Uncertainty Come in Quantum Mechanics " where I mention how does uncertainty work.

\section{Reference}

[1] Yasin ,M . Quantum Certainty Mechanics (2021) osf.oi

[2] Ozawa, M: Heisenberg's Original Derivation of the Uncertainty Principle and its Universally Valid Reformulations (2015).arXiv:1507.02010

[3] Busch , P: Heisenberg's uncertainty principle (2007). arXiv:quant-ph/0609185v3

[4] W. Heisenberg, Remarks on the origin of the relations of uncertainty, in: W. Price, S. Chissick(Eds.), The Uncertainty Principle and Foundations of Quantum Mechanics. A Fifty Years'Survey, J. Wiley \& Sons, London, 1977, pp. 3-6.

[5] W. Heisenberg, Uber den anschaulichen Inhalt der quantentheoretischen Ki " nematik und Mechanik, Z. Phys. 43 (1927) 172-198. 
[6] P. Busch, C.R. Shilladay, Complementarity and uncertainty in Mach-Zehnder interferometry and beyond, Phys. Rep. 435 (2006) 1-31.

[7] Planck M 1900 Zur Theorie des Gesetzes der Energieverteilung im NormalspectrumVerhandlungen der Deutschen Physikalischen Gesellschaft2, 237-45,English translation by D. ter Haar 1967The Old Quantum Theory(PergamonPress]:

[8] https://en.wikipedia.org/wiki/Uncertainty_principle.

[9] Heisenberg, W. The physical content of quantum kinematics and mechanics. In Wheeler, J. A. \& Zurek, W. H. (eds.) Quantum Theory and Measurement, 62-84 (Princeton UP, Princeton, NJ.

[10]Ozawa, M. Noise and disturbance in quantum measurements and operations. Proc. SPIE 6244, 62440Q (2006). 